\title{
A Novel Circular RNA hsa_circ_20403 Predicts Poor Prognosis and Promotes Progression in Osteosarcoma
}

\section{Rui Li (D 3222872742@qq.com )}

Chengde Medical University Affiliated Hospital

\section{Renzan Zhang}

Chengde Medical University Affiliated Hospital

Zhengpeng Liu

Chengde Medical University Affiliated Hospital

Yilong Zhang

Chengde Medical University Affiliated Hospital

\section{Research}

Keywords: Circular RNA, hsa_circ_20403, osteosarcoma, Prognosis

Posted Date: September 4th, 2020

DOI: https://doi.org/10.21203/rs.3.rs-61032/v1

License: (c) (i) This work is licensed under a Creative Commons Attribution 4.0 International License. Read Full License 


\section{Abstract}

Objective Osteosarcoma (OS) is the most frequent bone cancer in humans. Growing evidence suggests that circular RNAs (circRNAs) exert essential roles in regulating cancer initiation and metastasis. Some research were used Illumina HiSeq to screens differentially expressed of circRNAs in OS and found that hsa_circ_20403 is highly expressed in OS.

Methods In this study, we investigated the clinical application value and biological function of hsa_circ_20403 in OS. We were performed to verified the biological functions by CCK-8, flow cytometry, and transwell experiments.

Results We found that the expression of circ_20403 is enhanced in both OS tissue specimens and cells. In addition, its expression is closely related to clinic opathological characteristics and prognosis. More importantly, circ_20403 was overexpressed and knocked down in OS cells. We found that circ_20403 can promote cell proliferation, migration and invasion, which can regulate the proportion of $\mathrm{G} 1$ and $\mathrm{S}$ phase cells.

Conclusion Taken together, our study for the first time revealed hsa_circ_20403 as a carcinogen to promote the progression of OS. Our study suggested that hsa_circ_20403 can be used to predict the prognosis of OS and it might be a potential target for OS therapy.

\section{Introduction}

Osteosarcoma (OS) is the most common bone cancer in children and adolescents and it has a poor clinical prognosis $(1,2)$. The conventional therapy efficacy of OS is limited, and the proliferation and invasion were rapidly, which results the overall survival rate is poor in patients. The 5 -year survival rate of patients with advanced and metastatic osteosarcoma is only $20 \%(3)$. Because of improvements in surgery plus radiotherapy and chemotherapy, the 5 -year survival rate has reached $70 \%-80 \%$ of patients with OS(4). However, the survival of patients is still dismal and approximately $40-50 \%$ of patients will develop metastases $(5,6)$. The molecular mechanisms that regulate the formation and progression of osteosarcoma remain largely unclear. Therefore, it is essential for developing new candidate molecules and improving clinical outcomes of patients with OS(7). CircRNAs are a class of endogenous RNAs and they are existed in all eukaryotic cells, which was composed by a closed loop(8). The loop structure was covalently with 3 '-and 5 '-ends, which plays a key role in both pathological and physiological processes $(9,10)$. CircRNAs can be specifically bound with muscleblind (MBL, red circle), pre-mRNA into a circRNA (circMbl), and other protein factors(11). Recent studies showed that circRNAs are involved in colorectal cancer, lung cancer, breast cancer and other human cancer(12-14). Increasing evidence suggests that abnormally expressed circRNAs act as tumor suppressive or oncogenes genes in various malignant tumor(15-18). However, the study of circRNAs in OS is still seldom reported and the development is still limited. 
There are few studies on the relationship between OS and hsa_circ_20403. Xi et al.(19) research firstly found that hsa_circ_20403 in osteosarcoma tissues was significantly higher than their adjacent tissue. Furthermore, we used quantitative reverse transcription-polymerase chain reaction (qRT-PCR) to confirm the upregulation of hsa_circ_20403 in OS tissues and cell lines. Moreover, hsa_circ_20403 may be a potential regulator of OS and may be a development of novel therapeutic strategies for OS.

\section{Materials And Methods \\ 2.1 Cell culture}

Normal human osteoblast cell hFOB 1.19 and Human osteosarcoma cell (HOS $\square 20 S \square S a O S-2 \square M G-63)$ were purchased from the American Type Culture Collection. And they were maintained in Roswell Park Memorial Institute (RPMI) 1640 (Gibco, CA, USA) supplemented with 10\% foetal bovine serum (FBS) (Sigma-Aldrich, New Jersey, USA) and $100 \mu \mathrm{g} / \mathrm{ml}$ penicillin-streptomycin (Genom Bio-pharmaceutical Tech, Hangzhou, China). All cells were placed in $5 \% \mathrm{CO}_{2}$ at $37^{\circ} \mathrm{C}$.

\subsection{Clinical specimens}

A total of Patients $(n=78)$ with osteosarcoma tissues samples and corresponding noncancerous tissues were obtained between 2009 and 2015 at our hospital. All enrolled patients who underwent surgical procedure in our hospital and they haven't received radiotherapy or chemotherapy. All enrolled patients were obtained the informed consent before the study. All specimens were preserved immediately into liquid nitrogen after surgical resection for qRT-PCR detection. These 78 patients included 49 males and 29 females with a mean age of 24 years. The tumors were staged according to the Enneking staging

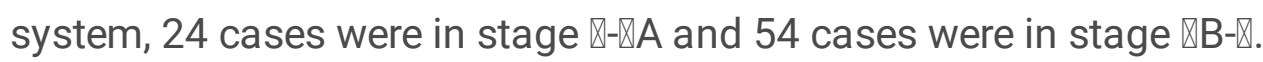

Inclusion criteria: 1) All patients did radical resection, and they were diagnosed with OS by postoperative pathological examination; 2) All patients did not receive radiotherapy and chemotherapy before operation; 3) All patients had complete clinical data and five year after the operation a clinical follow-up was performed. Exclusion criteria: 1) patients were non-primary osteosarcoma; 2) patients had cognitive dysfunction and other mental illness; 3 ) patients were accompanied by other primary tumors; 4) patients had lost to follow-up or incomplete clinical data. The project was authorized by the Clinical Research Ethics Committees of our Hospital.

\subsection{Next generation RNA sequencing analysis and Transfection}

The RNA-seq on ribosomal RNA-depleted total RNA for identifying circRNAs in osteosarcoma tissues samples, corresponding noncancerous tissues(19), and osteosarcoma cell is shown(20). HOS and SaOS2 cell transfection were carried out using a Lipofectamine 2000 liposome transfection kit (Takara, Shiga, Japan) according to the manufacturer's protocol and cell were observed $48 \mathrm{~h}$ at $37^{\circ} \mathrm{C}$ after transfection. HOS cell were divided into the following three treatment groups: Vector group, Over-circ group and mock 
group. SaOS-2 cell were divided into four treatment groups: si-NC, si-circ\#1 group, si-circ\#2 group and mock group. Mock group was without transfection. hsa_circ_20403 overexpression vector (over-circ), empty vector (vector), small interfering RNAs (siRNAs) specifically targeting hsa_circ_20403 (si-circ\#1 group and si-circ\#2) and a scramble RNA negative control (si-NC) were purchased from Sangon Biotech (Shanghai, China). The targeted sequence of si-RNA for hsa_circ_20403 (si-circ\#1 and si-circ\#2) was 5'AATCCAGAGTGAGCGATTACA-3' and 5'-ACGUGACACGUUCGGAGAATT-3'.

\subsection{RNA isolation and quantitative real-time PCR (qRT-PCR)}

Total RNA was isolated from hFOB1.19, HOS, U2OS, SaOS-2 and MG-63 cells, tissues or corresponding noncancerous tissues by RNeasy Mini Kit (Ambion, Texas, USA) according to the manufacturer's instructions. RNase $\mathrm{R}$ treatment was processed for $10 \mathrm{~min}$ at $37^{\circ} \mathrm{C}$ using $3 \mathrm{U} / \mathrm{mg}$ RNase R (Epicenter, WI, USA). Then CDNA was synthesized by reverse transcription, and fluorescence quanititavie dectection of the target gene was performed afterwards. All reactions were performed in a thermal cycler (ABI7500, $A B I$, USA) with primers (Sangon Biotech, Shanghai, China) for GADPH (glyceraldehyde-3-phosphate dehydrogenase) forward primers: 5'- GGC TGC CCA GAA CAT CAT - 3' and reverse: 5'- CGG ACA CAT TGG GGG TAG - 3'; hsa_circ_20403 forward primers: 5'- CTT TAC CCA GAAA TAC CCA C-3' and reverse primers: 5'- CCC TTA GGA TAG ACC TGT TG -3'. The qRT-PCR results were analyzed, that is, the cycle threshold (CT) values of each sample were analyzed, and calculated by the $2^{-\triangle \triangle C T}$ values of each target gene, and relative gene expression of hsa_circ_20403 were normalized to GAPDH.

HOS and Saos-2 cells were seeded in 6-well plates at $4 \times 10^{5}$ cells/well and maintained for $24 \mathrm{~h}$. HOS cell after transfection were divided into the following three treatment groups: Vector group, Over-circ group and mock group. SaOS-2 cell after transfection were divided into four treatment groups: si-NC, si-circ\#1 group, si-circ\#2 group and mock group. The total RNA of cells were the same extracted as above. All reactions were performed in a thermal cycler with primers (Sangon Biotech, Shanghai, China) for GADPH and hsa_circ_20403. The results of qRT-PCR were same analyzed as above.

\subsection{Cell Counting Kit-8 (CCK-8) assay}

Cell proliferation was determined by the CCK-8 (Dojindo, Japan) proliferation kits according to the manufacturer's instructions. HOS and Saos-2 cells after transfection were seeded in 96-well plates at $1 \times$ $10^{4}$ cells/well and cell viability evaluated after incubation for $24 \mathrm{~h}, 48 \mathrm{~h}, 72 \mathrm{~h}$ and $96 \mathrm{~h}$. CCK-8 solution $(10 \mu l)$ was added to each well and cells were incubated at $37^{\circ} \mathrm{C}$ for $2 \mathrm{~h}$. The absorbance at $450 \mathrm{~nm}$ was evaluated using a microplate reader.

\subsection{Flow Cytometry Analysis}

HOS and Saos- 2 cells were harvested 48 hours after transfection and clarified by low speed centrifugation ( $1200 \mathrm{r} / \mathrm{min}, 4 \mathrm{~min})$ at $4{ }^{\circ} \mathrm{C}$, incubated in $1 \mathrm{ml} 70 \%$ ethanol at $4{ }^{\circ} \mathrm{C}$ for $24 \mathrm{~h}$. They were then suspended in RNase A (Sigma-Aldrich Chemie $\mathrm{GmbH})(250 \mu \mathrm{g} / \mathrm{mL}$ ) and PI (Sigma-Aldrich Chemie $\mathrm{GmbH})(50 \mu \mathrm{g} / \mathrm{mL})$. After mixing at $37^{\circ} \mathrm{C}$ for 30 minutes in the dark, the cell cycle was measured with flow cytometry (FACScan, BD Biosciences). 


\subsection{Transwell assays}

Transwell chambers with 24-well $8 \mu \mathrm{m}$ pore polycarbonate filters (Corning Company, USA) were used in cell migration and invasion assays. For the invasion assay, $50 \mu \mathrm{L}$ of Matrigel at $300 \mu \mathrm{g} / \mathrm{mL}$ was added an insert and dried in a $37^{\circ} \mathrm{C}$ incubator for 1 hour to form a thin gel layer. HOS and Saos-2 cells were harvested 48 hours after transfection, HOS cell divided into vector group, Over-circ group and mock group. SaOS-2 divided into si-NC, si-circ\#1 group, si-circ\#2 group and mock group. The cells were collected and resuspended with serum-free PRMI 1640 and were adjusted to $1 \times 10^{5}$ cells $/ \mathrm{mL}$. After $24 \mathrm{~h}$ incubation, Matrigel and cells on the upper side of the membrane were wiped off, and the membrane were fixed in $4 \%$ paraformaldehyde in PBS at room temperature for $10 \mathrm{~min}$. Data were expressed as the average number of cells per insert.

For migration analysis, osteosarcoma cells $\left(1 \times 10^{5}\right.$ cells $\left./ \mathrm{mL}\right)$ were seeded in the upper chamber of a 24well transwell chambers. The upper chamber contained serum-free 1640 medium and the lower compartment contained medium with $10 \%$ FBS. The procedure was the same with invasion assay.

\subsection{Statistical analysis}

Statistical analyses were performed using SPSS 22.0 software (Chicago, IL). Data were expressed with mean \pm SD. Group differences were performed using one-way analysis of variance (ANOVA), Two-way RM ANOVA or Student paired $t$ test,Bonferroni's multiple comparisons test and Tukey's multiple comparisons were used for post inspection. Survival analysis was performed using Kaplan-Meier analysis with logrank test. Univariate and multivariate Cox analyses was using analysis the independent factors of prognosis. $P<0.05$ was considered significant.

\section{Results}

\section{1 hsa_circ_20403 is overexpressed in osteosarcoma tissues and cells}

qRT-PCR experiments were induced to investigate hsa_circ_20403 expression in 115 osteosarcoma tissues samples and corresponding noncancerous tissues. The results indicated that hsa_circ_20403 in osteosarcoma tissues were significantly higher than their adjacent tissue (Figure. 1A). In addition,

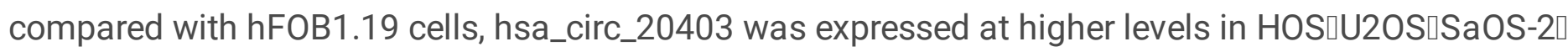
MG-63 cells (Figure. 1B). Because the expression of hsa_circ_20403 was the lowest and highest in HOS and SaOS-2 cells, therefor, in subsequent experiments, overexpression of hsa_circ_20403 was observed in HOS cells and knockdown of hsa_circ_20403 expression in Saos-2 cells.

\subsection{Relationship between hsa_circ_20403 expression and severity of OS}


78 patients with OS were classified into two groups by the median of hsa_circ_20403 expression: High expression of hsa_circ_20403 group $(n=37)$ and low hsa_circ_20403 group $(n=41)$. As shown in Table 1 , the clinical data of the 78 OS patients, we found that hsa_circ_20403 expression is related to Enneking stage and Tumor size, suggesting that hsa_circ_20403 can promote cancer progression and worsening. In addition, hsa_circ_20403 expression is irrelevant to age, gender, tumor site and histological grade.

Table 1

Association between hsa_circ_20403 expression and clinicopathological characteristics of patients with OS

\begin{tabular}{|c|c|c|c|c|}
\hline \multirow[b]{2}{*}{ Clinicopathologic characteristics } & \multicolumn{4}{|c|}{ circ_20403 expression } \\
\hline & $n$ & high & low & $P$ \\
\hline Age(years) & & & & 0.891 \\
\hline$\geqq 25$ & 31 & 15 & 16 & \\
\hline$<25$ & 47 & 22 & 25 & \\
\hline Gender & & & & 0.196 \\
\hline Female & 29 & 11 & 18 & \\
\hline Male & 49 & 26 & 23 & \\
\hline Enneking stage & & & & 0.031 \\
\hline$\bigotimes-\bigotimes A$ & 24 & 7 & 17 & \\
\hline$\varangle \mathrm{B}-\mathbb{\square}$ & 54 & 30 & 24 & \\
\hline Tumor site & & & & 0.107 \\
\hline Femur/Tibia & 61 & 26 & 35 & \\
\hline Other & 17 & 11 & 6 & \\
\hline Histological grade & & & & 0.465 \\
\hline Poorly/undifferentiated & 35 & 15 & 20 & \\
\hline Well/moderately & 43 & 22 & 21 & \\
\hline Tumor size(cm) & & & & $<0.001$ \\
\hline$\geqq 6$ & 36 & 28 & 8 & \\
\hline$<6$ & 42 & 9 & 33 & \\
\hline
\end{tabular}

\section{3 high-expression of circ_20403 was correlated with poor clinical outcomes}


Furthermore, we analysed the relationship between hsa_circ_20403 expression and prognosis. KaplanMeier survival analysis showed that the patients with high-expression of circ_20403 had a shorter survival time than patients with low-expression of circ_20403(Figure.2). Single-factor Cox showed that Enneking stage, Histological grade, Tumor size, and circ_20403 expression were significant differences in OS patients. The multivariate Cox regression analysis showed that Enneking stage and circ_20403 expression was an independent predictor of prognosis in patients with OS(Table 2).

Table 2

Factors affecting the prognosis of OS by univariate and multivariate Cox regression analyses

\begin{tabular}{|c|c|c|c|c|c|}
\hline \multirow[t]{2}{*}{ Variables } & & \multicolumn{2}{|c|}{ Univariate analysis } & \multicolumn{2}{|c|}{ Multivariate analysis } \\
\hline & & $\mathrm{HR}(95 \% \mathrm{Cl})$ & $P$ & $\mathrm{HR}(95 \% \mathrm{Cl})$ & $P$ \\
\hline Age(years) & $<25 \mathrm{vs} \geqq 25$ & $\begin{array}{l}0.872(0.667- \\
1.325)\end{array}$ & 0.711 & & \\
\hline Gender & Female vs Male & $\begin{array}{l}1.125(0.734- \\
1.552)\end{array}$ & 0.321 & & \\
\hline $\begin{array}{l}\text { Enneking } \\
\text { stage }\end{array}$ & $\rrbracket-\bigotimes A$ vs $₫ B-\bigotimes$ & $\begin{array}{l}3.779(1.908- \\
4.487)\end{array}$ & $<.001$ & $\begin{array}{l}2.165(1.341- \\
3.462)\end{array}$ & $\begin{array}{l}<.001 \\
0.00\end{array}$ \\
\hline Tumor site & Femur/Tibia vs Other & $\begin{array}{l}1.309(0.879- \\
1.833)\end{array}$ & 0.199 & & \\
\hline $\begin{array}{l}\text { Histological } \\
\text { grade }\end{array}$ & $\begin{array}{l}\text { Poorly/undifferentiated vs } \\
\text { Well/moderately }\end{array}$ & $\begin{array}{l}1.462(1.120- \\
1.766)\end{array}$ & 0.034 & $\begin{array}{l}1.165(0.794- \\
1.542)\end{array}$ & 0.201 \\
\hline $\begin{array}{l}\text { Tumor } \\
\text { size }(\mathrm{cm})\end{array}$ & $<6 \mathrm{vs} \geqq 6$ & $\begin{array}{l}1.820(1.093- \\
2.617)\end{array}$ & 0.003 & $\begin{array}{l}1.333(0.880- \\
1.905)\end{array}$ & 0.104 \\
\hline $\begin{array}{l}\text { circ_20403 } \\
\text { expression }\end{array}$ & low vs high & $\begin{array}{l}2.681(1.672- \\
3.190)\end{array}$ & $<.001$ & $\begin{array}{l}2.093(1.281- \\
2.986)\end{array}$ & $\begin{array}{l}<.001 \\
0.00\end{array}$ \\
\hline
\end{tabular}

To investigate the biological function of hsa_circ_20403 on osteosarcoma cell, our qRT-PCR results showed that the hsa_circ_20403 expression in the over-circ group was significantly higher than the vector group and mock group in HOS cell and the level of hsa_circ_20403 in the si-circ\#1 group and si-circ\#2 group were lower than si-NC group and mock group in SaOS-2 cell $(P<0.05$, Fig. 3A). The CCK-8 results showed that the proliferation ability of over-circ group was significantly enhanced than vector group in HOS cell. Compared with si-NC group, cell proliferation was decreased in si-circ\#1 group and si-circ\#2 group ( $P<0.05$, Fig. 3B). In addition, compare with the vector group, the proportion of G0/G1 phase cells significantly decrease in over-circ group of HOS cell, which was accompanied by a increase in the cell proportion in S phase. On the contrary, compare with the si-NC group, the proportion of G0/G1 phase cells significantly increase in si-circ\#1 group and si-circ\#2 group of SaOS-2 cell, which was accompanied by a decrease in the cell proportion in S phase. $(P<0.05$, Fig. $3 C)$. Similarly, we assessed the migration and 
invasion capability of HOS and SaOS-2 cells after transfection. Compare with the vector group, the results showed that the migration and invasion ability was obviously increased in over-circ group of HOS cell, while compare with the si-NC group, si-circ\#1 group and si-circ\#2 group markedly suppressed the migration and invasion ability in SaOS-2 cell $(P<0.05$, Fig. 3D, E).

\section{Discussion}

Circular RNAs (circRNAs) are important regulators of various biological processes. More and more circRNAs have been reported in many human malignancies, such as breast cancer, lung cancer, gastric cancer, and liver cancer (12-14,21-25). Recent research show that circRNAs could regulates OS cell proliferation, migration, and invasion(26,27). Circ-RNA-LARP4 might function as a sponge for miR-424, and miR-424 promoted proliferation and invasion of gastric cancer cells by targeting LATS1 gene(2). As a competitive RNA, circular RNA MYLK can promote the progression of bladder cancer by regulating vascular endothelial growth factor (VEGF) signaling pathway(28). Zhu et al.(25) found that the expression of circ-08001 was up-regulated in OS tissue and serum, and the higher expression of circ08001 was associated with poor prognosis of OS patients. The aim of this study was to investigate the expression of hsa_circ_20403 in osteosarcoma, and the relationship with clinicopathological characteristics and prognosis. Futhermore, knockdown and overexpression of circ_20403 reversed the proliferation and metastasis of osteosarcoma cells. At present, the relationship between OS and hsa_circ_20403 has rarely been reported. Xi et al.(19) assessed by bioinformatics analysis the differentially expression of circRNAs in osteosarcoma tissues and adjacent tissues of 13 patients, and they found that hsa_circ_20403 is highly expressed in osteosarcoma tissue, but its relationship with the prognosis of osteosarcoma and its biological functions has yet been reported.

We found that hsa_circ_20403 in osteosarcoma tissues was significantly higher than their adjacent tissue by qRT-PCR and Kaplan-Meier survival analysis showed that the patients with high-expression of circ_20403 had a shorter survival time than patients with low-expression of circ_20403. Then, we further found that compared with hFOB1.19 cells, hsa_circ_20403 was expressed at higher levels in HOS, U2OS, SaOS-2 and MG-63 cells. Further, Kaplan-Meier survival analysis showed that the survival time of patients with high-expression of circ_20403 is shorter than low-expression of circ_20403. Besides, Single-factor Cox found the prognostic indicators in OS patients and Enneking stage, Histological grade, Tumor size, and circ_20403 expression were significant differences in OS patients and the multivariate Cox regression analysis showed that Enneking stage and circ_20403 expression was an independent predictor of prognosis in patients with OS. This indicates that high-expression of circ_20403 can promote the progression of osteosarcoma, and it was correlated with poor clinical outcomes.

For biological function of hsa_circ_20403 on osteosarcoma cell, we found over-circ group remarkably promoted proliferation, migration, invasion, increase the cell proportion in $\mathrm{S}$ phase and decrease the cell proportion in G0/G1 phase of HOS cells. Conversely, knockdown of hsa_circ_20403 expression markedly suppressed proliferation, migration, invasion, decrease the cell proportion in $\mathrm{S}$ phase and increase the cell proportion in G0/G1 phase of Saos-2 cells. 
So far, many studies found that the deregulated circRNAs was related to the occurrence and development of OS. For example, circ_0000502 might promote the proliferation of MG63 and U2OS cells(29). Wu et al. revealed that overexpressed circ_0002052 can suppress the progression of OS through Wnt/ $\beta$-catenin signaling(30). Zhu et al. found that hsa_circ_0081001 was up-regulated in OS cell lines, which correlated with poor prognosis and it may be as a new diagnosis and treatment target(25). A previous study found that hsa_circ_0001564 was overexpressed in OS tissue and it maybe act as a potential biomarker for OS(31). Although hsa_circ_20403 independently predicts poor prognosis and promotes progression in osteosarcoma, the regulatory mechanism is still unknown. Thus, we will further explore the regulatory mechanism of hsa_circ_20403 in OS.

In summary, the study identified that a novel circRNA, hsa_circ_20403, was up-regulated in OS cell lines and tissues, correlated with poor prognosis and clinic opathological characteristics. In addition, preliminary results indicated that hsa_circ_20403 expression level may dynamically reflect the condition changes of OS patients. Our findings hsa_circ_20403 might be as a potential diagnosis and treatment target for OS.

\section{Conclusion}

Our study revealed hsa_circ_20403 as a carcinogen to promote the progression of OS for the first time. Our study suggested that hsa_circ_20403 can be used to predict the prognosis of OS and it might be a potential target for OS therapy.

\section{Abbreviations}

OS

Osteosarcoma

circRNAs

circular RNAs

MBL

muscleblind

qRT-PCR

quantitative reverse transcription-polymerase chain reaction

RPMI

Roswell Park Memorial Institute

FBS

foetal bovine serum

GADPH

glyceraldehyde-3-phosphate dehydrogenase

CCK-8

Cell Counting Kit-8

VEGF 
vascular endothelial growth factor

\section{Declarations}

Ethical Approval and Consent to participate

Not applicable

Consent for publication

Not applicable

Availability of supporting data

The datasets are available under reasonable request.

Competing interests

Not applicable

Funding

Not applicable

Authors' contributions

Not applicable

Acknowledgements

Not applicable

Authors' information

Rui Li,Renzan Zhang,Zhengpeng Liu,Yilong Zhang

\section{Figures}



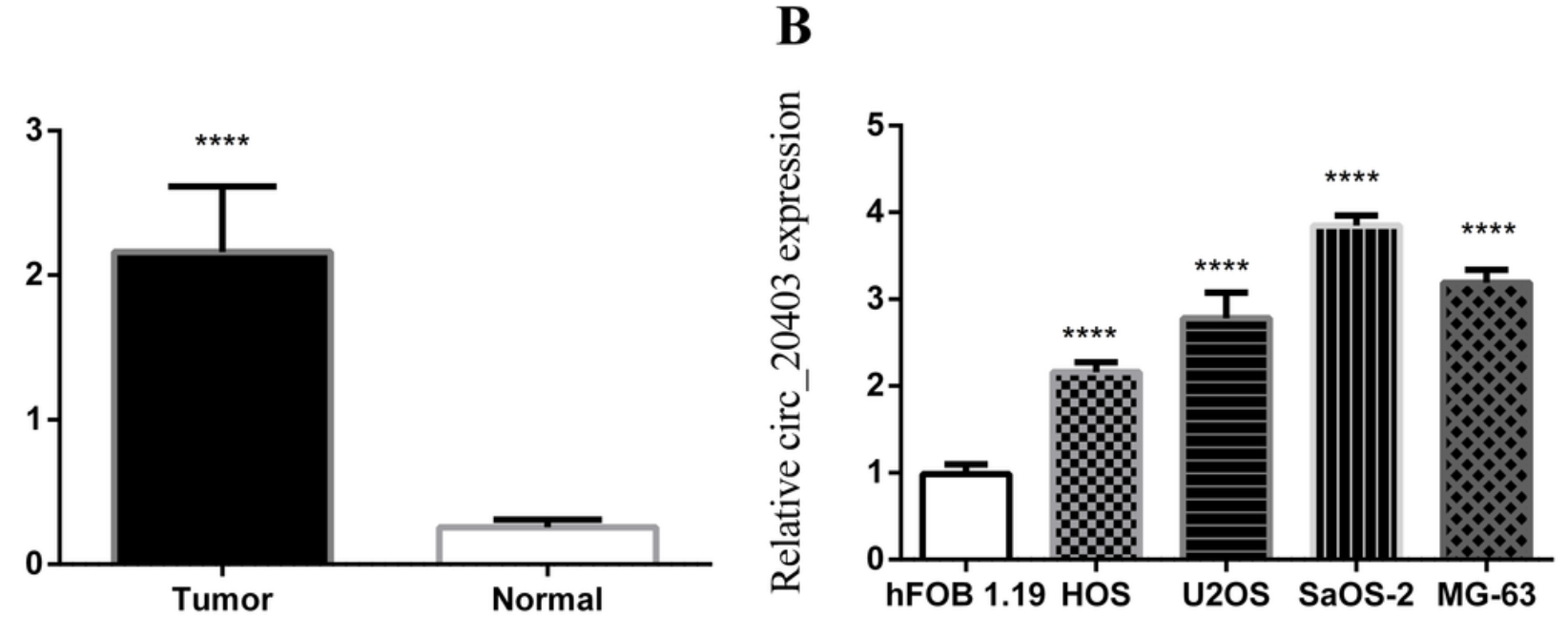

Figure 1

hsa_circ_20403 was highly expressed in OS cells and tissues. A. qRT-PCR showed that the expression of hsa_circ_20403 was significantly higher in OS tissues than para-carcinoma tissues $(n=78, t=37.97$, $\mathrm{P}<0.0001)$. B. Detection of differences in hsa_circ_20403 expression in normal osteoblasts (hFOB 1.19) and osteosarcoma cell lines (HOSロU2OS $\square$ SaOS-2and MG-63) by qRT-PCR $(n=3$ \ANAVO, $F(4,10)=119.6$, $\mathrm{P}<0.0001$, Dunnett's multiple comparisons test, $\mathrm{P}<0.0001)$.

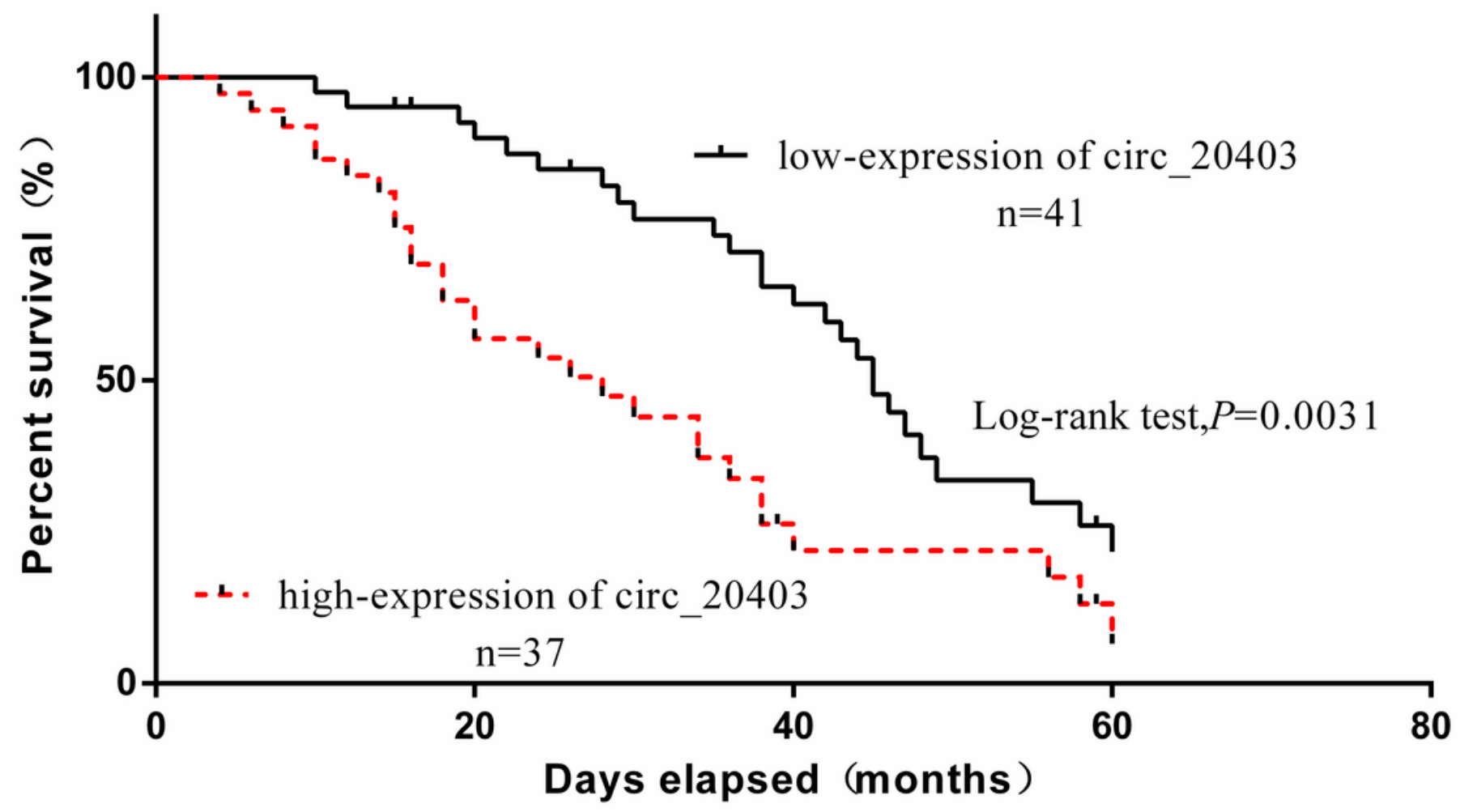

Figure 2 
The relationship between the expression of circ_20403 and the overall survival of patients with osteosarcoma. The log-rank test and Kaplan-Meier method were used to compare the overall survival between the high-circ_20403 expression group and the low-circ_20403 expression group. ( Chi square $=8.775 \otimes P=0.0031)$.
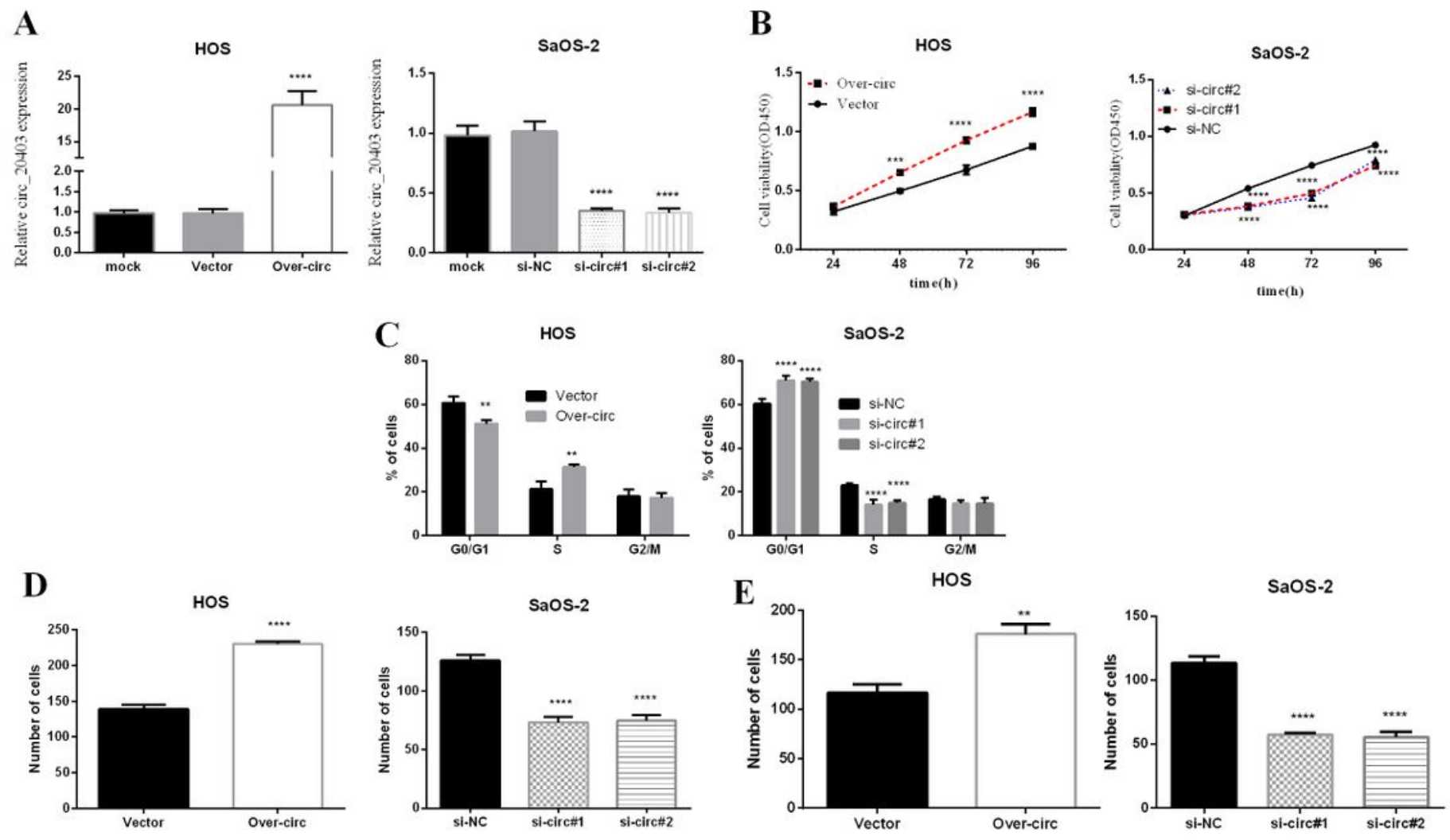

Figure 3

hsa_circ_20403 promotes the progression of osteosarcoma cell in vitro. A, The expression of circ_20403 was detected by qRT-PCR after transfection. Data were analyzed using one-way ANOVA test with Tukey's multiple comparisons. ( ${ }^{* \star * * P<0.0001)}$. B, Cell proliferation was determined by CCK-8 after transfection. Two-way RM ANOVA, Bonferroni's multiple comparisons test and Tukey's multiple comparisons test were used. ( $\left.{ }^{* *} P<0.001, * * \star * P<0.0001\right)$. C, Flow cytometry analysis was detected of cell cycle in HOS and SaOS-2 cell after transfection. Two-way RM ANOVA,Bonferroni's multiple comparisons test and Tukey's multiple comparisons test were used. ( $\left.{ }^{* \star *} P<0.001,{ }^{* \star * *} P<0.0001\right)$. D, Transwell assay was used to detect the migration and invasion of HOS and SaOS-2 cell after transfection. Data were analyzed by Unpaired $\mathrm{t}$ test, one-way ANOVA and Bonferroni's multiple comparisons test $\left(* * P<0.01,{ }^{* \star \star * P}<0.0001\right) . n=3$. 